\title{
Doing a leisure activity because there is nothing else to do: Related outcomes and intervention effects for adolescents
}

Mojdeh Motamedi ${ }^{a}$, Linda L. Caldwellb,c, Elisabeth H. Weybright ${ }^{d}$, Damon Jonese, Lisa Wegnerf ${ }^{\mathrm{f}}$, and Edward A. Smith ${ }^{c, e}$

aDepartment of Psychology, Pennsylvania State University; bDepartment of Recreation, Park, and Tourism Management, Pennsylvania State University; ${ }^{c}$ Faculty of Community and Health Sciences, University of the Western Cape; dDepartment of Human Development, Washington State University; eEdna Bennett Pierce

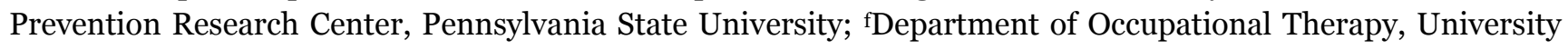
of the Western Cape

\begin{abstract}
This study examined whether a leisure-focused intervention, HealthWise, was related to reduced youth polysubstance use and delayed sexual debut via reducing how often youth did leisure activities because there was nothing else to do. HealthWise was compared to a nointervention control for 5,610 high school students from eighth to tenth grades in townships near Cape Town, South Africa. Three specific leisure activities were examined: spending time with friends, playing sports, and going to parks. Among girls, spending time with friends because there was nothing else to do significantly mediated the effect of HealthWise on reducing frequent polysubstance use in the past month. For boys, spending time in parks because there was nothing else to do mediated the effect of HealthWise on delayed sexual debut. Results partially supported the HealthWise logic model of impacting risky behaviors via leisure and the value of prevention programs addressing the reasons behind leisure choices.
\end{abstract}

Keywords: Adolescence; leisure activity; sexual debut; South Africa; substance use

It is widely accepted that leisure is one of the most important contexts in which youth development can occur. At the same time, however, the leisure context is home to negative behaviors (e.g., substance use, early sex). The mechanisms by which such behaviors occur in leisure are not well understood. To further an understanding of the pathways of positive and negative behavior in leisure, we focus on three common adolescent leisure activities: spending time with friends, playing sports, and going to parks. These activities are protective in some situations (e.g., contexts for developing social support, a sense of belonging and selfconfidence) and risky in others (e.g., contexts for negative peer pressure; Fredricks \& Eccles, 2005). 
One way to identify whether leisure activity choices of youth may be risky or protective is to better understand the reasoning behind those choices. We use an "action-incontext" perspective (Silbereisen, Eyferth, \& Rudinger, 1986) that focuses on the interaction of the leisure activity, context, and experience (LACE model; Caldwell, \& Faulk, 2013) to explain the reasoning behind activity choices of youth. This perspective suggests that it is important to understand whether the actions of youth are self-determined (i.e., internally compelled, e.g., "I want to") or externally compelled (e.g., "I have to") versus done absent of either internal or external compulsion (i.e., amotivation, e.g., "there is nothing else to do). Youth who typically engage in self-determined activities have better health-related outcomes (e.g., improved smoking abstinence, positive affect, quality of life, and exercise) whereas externally driven activities and amotivated behaviors are related to negative outcomes (e.g., greater depression and anxiety; Ng et al., 2012).

Related to amotivation, engaging in a leisure activity because there is nothing else to do suggests a lack of optimal arousal that could lead to risky sensation-seeking behaviors (Caldwell et al., 1999) and is an especially common experience in underresourced, lowincome contexts such as the urban townships of Cape Town, South Africa (SA), which is the context for our study. According to youth focus groups from this area, two of the four most common leisure themes youth reported are having limited leisure resources and opportunities in the community and engaging in risky behavior because there is nothing else to do (Wegner, 2011). Factors that contribute to this sense of nothing else to do, such as boredom, amotivation, and a perceived lack of community activities, have all been linked to risky behaviors such as substance use and dropping out of school (Caldwell, Bradley, \& Coffman, 2009; Motamedi, Caldwell, Wegner, Smith, \& Jones, 2016; Wegner, Flisher, Chikobvu, Lombard, \& King, 2008; Weybright, Caldwell, Ram, Smith, \& Wegner, 2015). Given the association between lower well-being and risky behaviors (e.g., Sheldon, Ryan, Deci, \& Kasser, 2004), this article focuses on NED activities, the time spent engaged in leisure activities because there is nothing else to do, in comparison to activities done for other reasons. To our knowledge, this is the first article that specifically connects this reason for leisure activities with specific activity types.

A gender by context interaction regarding leisure activity participation may also play a role in when leisure activities are linked to risky behaviors. Gender differences may be associated with reasons for leisure activity choices, perceptions of available activities affecting risky behavior choices, and participation in various activities differentially linking to risky behaviors (e.g., sports are linked to more risk taking for adolescent boys but not girls; Caldwell, Patrick, Smith, Palen, \& Wegner, 2010; Kaufman, Clark, Manzini, \& May, 2004; Motamedi et al., 2016). In addition, although both boys and girls in the same area as this study report a lack of safe leisure opportunities as a major constraint to participation in leisure activities, girls also face a major constraint of gender norms (Palen et al, 2010). This suggests that how youth experience their community and available leisure activities varies by gender, which may be linked to differences in their risky behavior rates.

Given contextual limitations on leisure choices and the related prevalence of feeling there is nothing to do in townships surrounding Cape Town, HealthWise South Africa: Life Skills for Young Adults (HealthWise) was developed, implemented, and evaluated. For details regarding the development of this intervention and how it was adapted for the local 
context, readers are directed to Caldwell et al. (2004). Going beyond other high schoolbased risk prevention programs that have focused primarily on refusal skills and parenting programs, HealthWise takes an innovative approach by adding a positive youth development framework to help youth develop a healthier and more selfregulated orientation toward participating in leisure activities (Franklin \& Corcoran, 2000; Skiba, Monroe, \& Wodarski, 2004). Specifically, HealthWise builds on other programs such as Botvin's evidence-based LifeSkills Training program by covering risky behavior norms and realities and refusal, relationship, and self-management skills (Botvin \& Griffin 2004). However, HealthWise is unique in that half of the curriculum also addresses leisure, reasons for activity choices, how to avoid boredom, and how to develop and engage in interesting community activities. High school teachers cover this curriculum across 12 lessons in eighth grade and six lessons in ninth grade as part of the nationally mandated Life Orientation curriculum.

As part of developing a more self-regulated orientation, HealthWise is intended to help youth learn to avoid boredom and risky behaviors by being more intentional in choosing leisure activities for pleasure or achieving a goal (Ryan \& Deci, 2000; Ryan, Patrick, Deci, \& Williams, 2008). This behavioral orientation may be especially important for avoiding risky behaviors when living in an area with a dearth of healthy leisure opportunities and a high prevalence of peers engaging in risky behaviors including substance use, violence, and early sex such as in the urban townships of Cape Town, SA (Reddy et al., 2010). Indeed, other research has shown that HealthWise reduces risky behaviors and boredom, but the change mechanisms by which this occurs have not yet been tested in a mediational model focused on reasons behind leisure activities (Caldwell et al. 2010; Smith et al. 2008; Tibbits, Smith, Caldwell, \& Flisher, 2011). Given findings that HealthWise affects sexual activity and polysubstance use (Tibbits et al., 2011), we focused on examining mediators for these outcomes. Specifically, we examined whether HealthWise reduced target NED activities and whether this in turn reduced frequent polysubstance use and the likelihood of sexual debut among youth. We also examined gender differences across these factors.

\section{Method}

\section{Study design}

HealthWise was trialed from 2004 to 2008 in a low-income, densely populated urban township in Mitchell's Plain (near Cape Town, SA). Four of 19 eligible secondary (high) schools were randomly assigned to the experimental condition where teachers taught HealthWise. Five comparison schools matched on socioeconomic and demographic characteristics with the HealthWise schools were selected as comparison schools to continue delivering the national Life Orientation curriculum as they typically would.

Data were collected through youth self-report surveys prior to the implementation of HealthWise at the start of eighth grade (baseline, when high school starts in South Africa) and at the start of 1oth grade (follow-up after the intervention ends in ninth grade). We longitudinally followed three different cohorts of students from eighth grade through 10th grade with one cohort (38.60\% of full sample) starting eighth grade in 2004, another (32.8\%) starting eighth grade in 2005, and a third (28.60\%) starting eighth grade in 2006. 
Table 1. Baseline descriptive statistics by sample.

\begin{tabular}{|c|c|c|c|c|c|c|}
\hline \multirow[b]{2}{*}{ Condition } & \multicolumn{3}{|c|}{ Full sample $(N=5,610)$} & \multicolumn{3}{|c|}{ Baseline virgins $(N=4,982 ; 89 \%)$} \\
\hline & $\begin{array}{c}\text { Control } \\
\% / \mathrm{M}(\mathrm{SD})\end{array}$ & $\begin{array}{c}\text { HealthWise } \\
\% / M(S D)\end{array}$ & $\mathrm{t} / \chi^{2}$ & $\begin{array}{c}\text { Control } \\
\% / \mathrm{M}(\mathrm{SD})\end{array}$ & $\begin{array}{c}\text { HealthWise } \\
\% / M(S D)\end{array}$ & $\mathrm{t} / \chi^{2}$ \\
\hline Male & $50.30 \%$ & $49.80 \%$ & 0.15 & $47.10 \%$ & $45.40 \%$ & 1.31 \\
\hline Age (12-19 years) & $13.92(0.78)$ & $14.06(0.88)$ & $-6.00^{* *}$ & $13.87(0.75)$ & $13.98(0.81)$ & $-4.62^{* *}$ \\
\hline Race $^{\mathrm{a}}$ & & & $90.71 * *$ & & & $73.89 * *$ \\
\hline Black & $5.85 \%$ & $13.12 \%$ & & $4.81 \%$ & $11.26 \%$ & \\
\hline White & $4.67 \%$ & $4.63 \%$ & & $4.52 \%$ & $4.32 \%$ & \\
\hline Mixed race & $88.35 \%$ & $80.89 \%$ & & $89.65 \%$ & $83.14 \%$ & \\
\hline Family income $(0-2)$ & $1.41(0.63)$ & $1.33(0.64)$ & $4.79 * *$ & $1.42(0.63)$ & $1.34(0.64)$ & $4.19^{* *}$ \\
\hline \multicolumn{7}{|l|}{ NED: } \\
\hline Hang with friends $(0-2)$ & $0.42(0.69)$ & $0.39(0.67)$ & 1.95 & $0.42(0.69)$ & $0.39(0.68)$ & 1.70 \\
\hline Do sports $(0-1)$ & $0.11(0.32)$ & $0.12(0.32)$ & 0.47 & $0.11(0.31)$ & $0.11(0.32)$ & 0.50 \\
\hline Go to parks $(0-2)$ & $0.17(0.49)$ & $0.17(0.49)$ & 0.00 & $0.17(0.48)$ & $0.17(0.49)$ & -0.11 \\
\hline Proportion of all NED activities (0-1) & $0.22(0.38)$ & $0.22(0.38)$ & 0.68 & $0.22(0.38)$ & $0.22(0.38)$ & 0.28 \\
\hline Polysubstance use (0-3) & $0.18(0.50)$ & $0.21(0.53)$ & -1.51 & $0.15(0.44)$ & $0.15(0.43)$ & -0.09 \\
\hline
\end{tabular}

Note. NED = the time spent engaged in a leisure activity because there is nothing else to do.

a $1 \%$ indicated other for any sample.

${ }^{*} p<.05 ;{ }^{* *} p<.01$ with Student's $t$ test for continuous variables and $\chi^{2}$ for categorical variables.

We chose to examine the NED activities of students at the beginning of 1oth grade, or after the first summer break postintervention, to understand the impact of HealthWise. This was to reflect the possible influence of HealthWise after a long period of time (summer break) when students may have been more prone to feeling they had nothing to do.

Across cohorts, there were 6,253 students. Analyses were based on two samples: the full sample of students with baseline data $(5,610)$ and a baseline virgins subsample $(4,982)$. As shown in Table 1, across the three samples, participants were evenly split on gender, on average 14 years old, and majority mixed race (historically labeled "Colored" under Apartheid in South Africa to refer to individuals with a mix of Asian, European, and African background; 80.89\%-89.65\%), with the rest identifying as Black (4.81\%-13.10\%), White (4.32\%-5.19\%), and Indian/other (approximately $1 \%$ for any sample, not shown). Owning a car or living in a brick house was used as a proxy for family income; the mean score was 1.38 on a 2-point scale for the entire sample, which suggests a majority of the families in this sample either had a car or lived in a brick house. At baseline, compared to the control group, HealthWise youth were older, more likely to be Black (or not mixed race), and from poorer families (see Table 1). This applied to all samples except for Cohort 3, where the two conditions did not have significantly different baseline family income.

\section{Measures}

\section{Leisure activities}

We assessed the combined role of type of leisure activity, time spent in activity, and reason for activity in two ways. First, we focused on three specific target activities done because there was nothing else to do. Youth were asked whether in the past 4 weeks they spent time 
doing each of the following activities after school/over weekends: sports or other physical activities, hanging out with friends, and going to a park or community/sports center (in South Africa, this includes vacant lots that frequently have gang/drug activity; herein abbreviated as "parks"). Those who said yes to spending time doing each activity were then asked how much time they spent doing each activity per week ( $0=$ less than 1 hour; $1=1-5$ hours; $2=6-10$ hours; $3=$ more than 10 hours) and chose from a set of reasons for why they usually did this activity (i.e., nothing else to do, have to, for a purpose, and want to). Second, we created a proportion variable combining the three NED activities to understand whether study effects were particular to a specific NED activity versus generally any NED activity.

NED activities. We created one composite variable per activity to reflect those who did the activity because there was nothing else to do versus those who did the activity for any other reason (i.e., have to, want to, or for a purpose) or did not do the activity. Measures were dior trichotomized to reflect the time spent in each activity because there was nothing else to do. The decision to di- or trichotomize was based on the distribution of data such that there was at least $5 \%$ of the sample in each category. Hanging out with friends because there was nothing else to do (NED with friends) was trichotomized as follows: $0=$ don't hang out with friends or do so for a reason other than nothing else to do $(71 \%$ of the full sample at baseline), 1 = hang with friends for 0 to under 10 hours per week for nothing else to do (18\%), and 2 = hang with friends for 10 or more hours per week for nothing else to do (11\%). Doing sports because there was nothing else to do (NED sports) was dichotomized as follows: $0=$ don't do sports or do so for a reason other than nothing else to do ( $89 \%$ of the full sample at baseline) and $1=$ do sports for any amount of time for nothing else to do (11\%). We trichotomized going to parks for nothing else to do (NED parks) as $0=$ don't go to parks or do so for a reason other than nothing else to do (88\%), 1 = go to parks for o to under 5 hours per week for nothing else to do (7\%), and $2=$ go to parks for 5 or more hours per week because there is nothing else to do (5\%). The correlation among the NED activities at both timepoints ranged from $\mathrm{r}=.08$ to $\mathrm{r}=.12$.

Proportion of NED activities. This proportion was created by summing all three activities done for more than 1 hour because there was nothing else to do and dividing by the sum of activities done for more than 1 hour a week for any reason. A cutoff of 1 hour per week was used to dichotomize each of these counts to capture sufficient activity involvement. For example, someone who goes to parks more than 1 hour a week due to having nothing else to do, but hangs out with friends and does sports, each for more than 1 hour a week for another reason (e.g., because that person wants to or has to), would have a proportion score of .33.

\section{Frequent polysubstance use in the past month}

Youth responded to a series of questions to assess their use of alcohol, tobacco, and marijuana. For each, youth were first asked if they had ever used the substances in their lives. Those who indicated use were then asked "During the past 4 weeks how many: alcoholic drinks did you have? ( $1=1$ or less, $2=2-3$ and $3=4$ or more), cigarettes did you smoke? ( $1=1$ or less, $2=2-9$, and $3=10$ or more) and times did you use dagga (marijuana)? 
( $1=1$ time, $2=2-3$ times, and $3=4$ or more times)." We used the method by Tibbits et al. (2011) for summarizing these variables to represent frequent past month polysubstance use. Youth who reported no lifetime or past month use of any given substance were coded as 0 . Those who reported past month use were coded as 0 if they indicated response choices 1 or 2 for amount used in the past 4weeks and coded as 1 if they indicated response choice 3, which was considered frequent use of the substance. Scores for each of the three substances were summed for their final score $(0=$ no frequent use of any substance, $1=$ frequent use of 1 substance, 2 = frequent use of 2 substances, and $3=$ frequent use of 3 substances).

\section{Sexual debut}

Sexual debut was assessed with the question "Have you ever had sex? This means intimate contact with someone during which the penis enters the vagina (female private parts)" ( $\mathrm{o}=$ no; 1 = yes).

\section{Family income}

As the income of caregivers often fluctuated with employment options and youth typically did not know the income of their caregivers, we created a proxy for income as a covariate based on a sum of two dichotomized items: "family owns a motor car" (o = no, $1=$ yes) and "which of the following best describes your home" (o = shack, wendy house/backyard dwelling, tent/traditional dwelling, or other, and $1=$ brick house, flat, or maisonette).

\section{Missing data}

There were some missing data within each assessment period for participants. Between the start and end of each semester, an average of $12 \%$ of the target population was lost due to attrition, for a total of up to $44 \%$ missing on measures by the start of 1 oth grade $\left(\mathrm{N}_{1 \text { oth grade start }}\right.$ $=3,126$ ). Those who did not have data by the start of 1oth grade were, at the start of eighth grade, older, poorer, more likely to be male, less likely to be mixed race, more likely to be using multiple substances frequently, and more likely to have had sex. Although this missingness is not at random (MNAR), simulation studies (Collins, Schafer, \& Kam, 2001) and analyses with this data set (Graham, Palen, Smith, \& Caldwell, 2008) have shown that any bias in estimates resulting from attrition rates as high as 50\% does not necessarily compromise the validity of the findings as long as appropriate missing data procedures are used (Collins et al., 2001; Graham et al., 2008). In addition, this missingness was likely not unique to this study as 6o\% of students drop out between first grade and the end of high school in South Africa and dropout characteristics are consistent with characteristics associated with attrition in this study (Townsend, Flisher, Chikobvu, Lombard, \& King, 2008; Weybright, Caldwell, Xie, Wegner, \& Smith, 2017). Thus, to account for missing data, all analyses used full information maximum likelihood with maximum likelihood estimation in Mplus using baseline characteristics (i.e., age and family income) to predict missingness. 


\section{Analytic strategy}

We sequentially ran path analyses starting with testing for direct intervention effects on risky behavior outcomes and then the hypothesized mediators. Next we used path analyses to assess whether doing NED activities at the start of 10th grade mediated the association between HealthWise and risky behaviors by the start of 1oth grade controlling for respective eighth-grade behaviors and NED activities. All analyses were separated by gender. For these analyses, HealthWise was dichotomized such that $1=$ students who received HealthWise and - $1=$ the no-intervention comparison group. For the outcome of frequent polysubstance use, the full sample was used. The sample was limited to baseline virgins for assessing the sexual debut. For each outcome and gender, we tested a model including all three NED activities as mediators and another separate model with the proportion of NED activities as a mediator. Since direct effects are not required for mediation (e.g., MacKinnon, Lockwood, Hoffman, West, \& Sheets, 2002), we examined indirect effects via hypothesized mediators based on the HealthWise logic model and theory (Caldwell et al., 2004) regardless of whether there were direct effects of HealthWise on outcomes. Given our focus on mediation and sample size, we only discuss direct effects significant at the $\mathrm{p}=.05$ value. Per recommendations by Muth en and Muth en (2010) for testing mediation with dichotomous outcomes, robust weighted least squares were used to estimate paths in Mplus.

Cohort and baseline characteristics corresponding to relevant mediators and outcomes were controlled for in all analyses. To control for baseline differences between the intervention and control condition, we also controlled for age and family income. We were unable to also control for race because adding it to our analytic models created high collinearity among the covariates, despite the control condition having more mixed-race students than the HealthWise condition. Instead, we ran all analyses with only mixed-race students and found the same pattern of findings described in the following when the analyses were restricted to students identifying as mixed race. Given the small sample size for the other race subgroups (e.g., our sample contained 5o White, female, baseline virgins at the start of 10th grade), we were underpowered for analyses with only White and only Black students. Nevertheless, the direction of the findings was similar across races.

\section{Results}

\section{Direct effects of HealthWise}

First, we assessed the direct effect of HealthWise on outcomes for girls and boys. HealthWise did not have significant direct effects on any of the outcomes (i.e., frequent polysubstance use and sexual debut among baseline virgins). Next, for each gender and outcome, we examined the effect of HealthWise on NED activities with all the activities in each model and separately for the proportion of NED activities. In Figures 1 and 2, we report either beta weights (if the dependent variable was trichotomized) or odds ratios (if the dependent variable was dichotomized).

For girls, HealthWise reduced NED with friends, NED sports, and the overall proportion of NED activities $(\mathrm{p}<.05)$ but did not affect NED parks. For boys, HealthWise 
reduced NED parks $(\mathrm{p}<.05)$ but did not affect NED with friends, NED sports, and the proportion of NED activities. These associations applied for both analyses with the full sample and analyses with the sample limited to baseline virgins.

\section{Mediation results}

As shown in Figures 1 and 2, the effect of HealthWise on each outcome was examined separately by gender with all NED activities included in each model as potential mediators, as well as in separate models for the proportion of NED activities. For the full sample, we looked at effects on frequent polysubstance use in the past month. Among girls, more NED with friends was associated with more frequent polysubstance use at the start of 1oth grade, controlling for eighth-grade levels of use. Furthermore, for girls, NED with friends at the start of 10th grade mediated the association between HealthWise and polysubstance use $(\mathrm{p}<.05)$ such that HealthWise girls had less NED with friends. The proportion of NED activities was not related to polysubstance use for girls. For boys, greater NED with friends and proportion of NED activities were related to more frequent polysubstance use $(\mathrm{p}<.05)$. However, there was no evidence of HealthWise having an influence on polysubstance use for boys through these paths. For both boys and girls, NED sports and NED parks were not associated with polysubstance use.
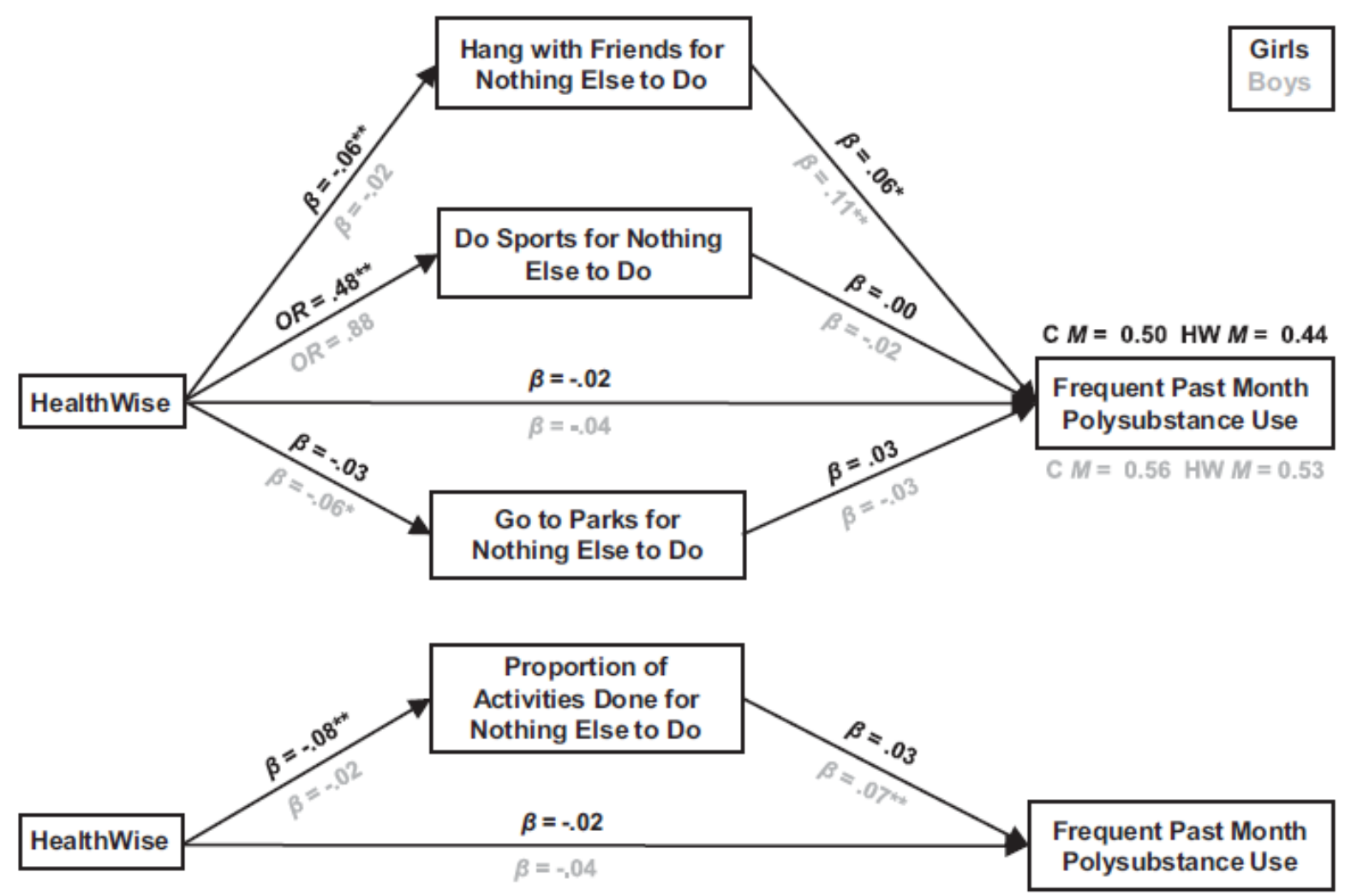

Figure. 1. Indirect effect of HealthWise on frequent past month polysubstance use for full sample with baseline data. $\mathrm{M}=$ mean; $\mathrm{C}=$ control; $\beta=$ Beta weight; $O R=$ odds ratio. Direct effect of treatment without mediators in the model was nonsignificant for polysubstance use (girls: $\beta=-.03$, $p=.24$; boys: $\beta=-.07, p=.14) .{ }^{*} p<.05 ;{ }^{*} p<.01$. 

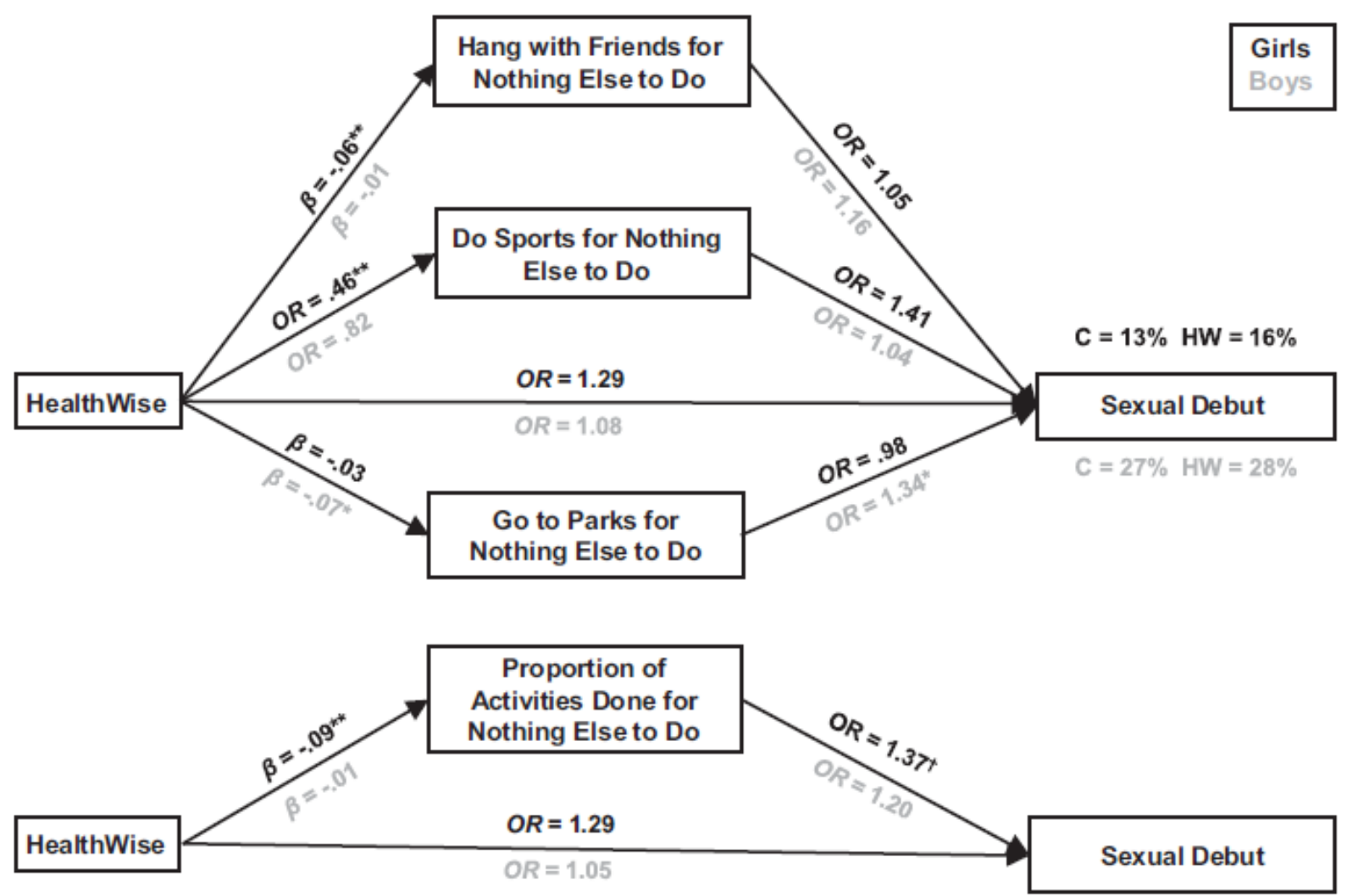

Figure. 2. Indirect effect of HealthWise on sexual debut for baseline virgins. $M=$ mean; $C=$ control; $\beta=$ Beta weight; $O R=$ odds ratio. Direct effect of treatment without mediators in the model was nonsignificant for sexual debut (girls: $O R: 1.29, p=.08$; boys: $O R: 1.06, p=.69$ ). ${ }^{*} p<.05$; ${ }^{* *} p<.01$.

Among baseline virgin girls, there were no significant associations among sexual debut and any of the NED activities or the proportion of NED activities. For baseline virgin boys, more NED parks was associated with earlier sexual debut and mediated the path between HealthWise and sexual debut $(\mathrm{p}<.05)$ such that HealthWise boys had less NED parks. NED with friends, NED sports, and the proportion of NED activities were not associated with sexual debut for baseline virgin boys.

\section{Discussion}

We conclude that to reduce risky behaviors for adolescents, it is important to consider the role of NED activities, which is a combination of the type of leisure activities and time spent in the activities because there is nothing else to do. Furthermore, a leisure focused intervention such as HealthWise is helpful in reducing risky behaviors among adolescents by helping them learn to adopt a more intentional approach to choosing leisure activities. It is also important to consider context, such as gender and accompanying gender norms, since our findings varied by gender. Specifically, HealthWise reduced NED with friends, NED sports, and the overall proportion of NED activities for girls but not boys. This is important, in part, because for girls and boys, NED with friends was associated with more frequent polysubstance use. In turn, among girls, HealthWise reduced frequent polysubstance use in the past month by reducing NED with friends. 
For boys, HealthWise reduced NED parks. In interpreting findings about the time spent in parks, it is important to keep in mind that in South Africa, parks includes reference to community centers and run-down or vacant lots known for gang/drug activity. By reducing NED parks for boys, HealthWise reduced the likelihood of their sexual debut by the start of 10th grade. In addition, although the proportion of NED activities was not a significant risk factor for polysubstance use and sexual debut among girls, having a higher proportion of NED activities was associated with more frequent polysubstance use among boys. These findings suggest that HealthWise promoted a more intentional perspective for boys to engage in when at parks, and for girls, to engage in sports, hang out with friends, and engage in activities generally. Thus, this study provides some insight into the mechanism by which risky behavior can be influenced by an intervention that focuses on healthy leisure and the reasons behind leisure choices.

The difference in findings with regard to specific activities versus the proportion of NED activities is notable. The findings highlight that sometimes the context of the activity may be less relevant and simply a high proportion of NED activities is risky, such as with boys and frequent polysubstance use. By contrast, the activity context matters for how frequently girls use multiple substances since their risk was only higher when they were engaged in NED with friends. These findings suggest that there is variation in when a specific NED activity is risky versus the overall proportion of NED activities and that this risk can vary by gender.

The importance of considering gender within a community context is highlighted by such gender differences, which may be due to a variety of reasons. For example, HealthWise may have specifically influenced what girls focus on in leisure interactions in comparison to what boys focus on. Specifically, the interpersonal interactions of girls typically focus more on prioritizing connection with others (e.g., spending time with friends) and how their peers evaluate them, while the interactions of boys focus more on status and dominance, potentially through physical activities such as sports (Rose \& Rudolph, 2006; Seiffge-Krenke, 2011). Thus, given the HealthWise focus on understanding the reasons behind activity choices, HealthWise may have played a role in girls evaluating their reasons for what they prioritize in their peer interactions, which, as these studies indicate, differs from how boys experience their peers. In addition, these gender differences may be related to boys often having more autonomy compared to girls to spend unsupervised time in areas such as parks, and thus, time spent in parks may be less of an option and less relevant for girls (Kaufman et al., 2004).

Some of the difference in the outcomes also may be related to differences in when girls and boys begin to engage in different risky behaviors. For example, HealthWise may delay sexual debut among girls older than those in this study since not many girls may have started having sex by the start of 1oth grade in comparison to boys in this sample regardless of the intervention condition (Tibbits et al., 2011). Last, these findings could also be partially due to girls learning how to find other, healthier leisure opportunities after going through the HealthWise program and not being as constrained by gender norms to feeling there is nothing else to do than hang out with friends (Motamedi et al., 2016). Overall, the reasons for these gender differences may be multifaceted, and more research is necessary to understand them. 
Such gender-specific findings are important given a review of Kumpfer, Smith, and Summerhays (2008) indicating there is a lack of prevention programs to specifically reduce substance use among girls in light of their increased substance use over the years in the United States. Our findings suggest addressing how doing specific leisure activities, and not necessarily a constellation of activities, because there is nothing else to do can help reduce related risky behaviors by gender. For example, it may be important for an intervention facilitator to specifically discuss the reasons for girls spending time with friends, the reasons for boys spending time at parks, and what other reasons and activities could be healthier for them to reach their goals and to address gender-specific pressures influencing their reasoning. These findings can also help us understand why sometimes one leisure activity, like hanging out with friends, can be risky in one environment

(e.g., hanging out with friends because there is nothing else to do among girls) but protective in another (e.g., being with friends to have a sense of social support, which reduces the likelihood of depressive symptoms; Shilubane et al., 2014).

Although we are not able to untangle whether these outcomes related to NED activities are due to boredom, amotivation, a lack of resources in the community, or something else, action-in-context (Silbereisen, Eyferth, \& Rudinger, 1986) and social cognitive (Bandura, 2001) theories would suggest the NED activities outcomes are a result of the reciprocal interaction of these factors, particularly in the context of this study. The confluence of these factors in low-income communities is especially important given the lack of resources and leisure opportunities. This study suggests leisure focused programs such as HealthWise can influence the typical activities of youth to reduce potential negative impacts related to the constraints and risk factors prevalent in these communities in a way that is relevant and feasible for them.

The International Narcotics Control Board (2013) and others suggest that preventive efforts to reduce youth drug misuse in marginalized societies, and by extension other risky behaviors, should include the provision of community-based interventions, including leisure opportunities. This perspective is important because most adolescent sex and substance use prevention programs tend to focus primarily on refusal skills, parenting, coping skills, decision making, and health education (Franklin \& Corcoran, 2000; Skiba et al., 2004), without a focus on leisure as an important context for life skill development. HealthWise is unique since in addition to using these approaches (e.g., giving equal attention to health education by addressing proper use of condoms and decision making around substance use and risky sex), HealthWise also equally addresses the health-promoting and protective factors of leisure (e.g., reasons behind leisure activity choices and avoiding leisure boredom).

Overall, our findings provide evidence that a main focus on leisure is a valuable perspective to add to risky behavior prevention programs. We would add to the advice of the International Narcotics Control Board, however, that it is not enough just to focus on improving community leisure opportunities. Young people need to be prepared to understand how their activity choices, as well as the reasons behind these choices, can lead to positive and healthy outcomes or negative and risky outcomes. Young people also need to learn specific skills for increasing self-determined and autonomous behaviour to avoid situations that lead to boredom and a behavioral orientation where they do leisure activities for unidentifiable reasons. Finally, young people need to understand how environmental 
factors (e.g., peer approval of an activity) may alter motives and how to appropriately respond (Fredricks \& Eccles, 2005).

\section{Limitations and future directions}

Given the importance of context and local norms, future studies should examine the role that reasons behind leisure activity choices play in risky behaviors in other populations. In South Africa, this is especially necessary among Black youth (the majority in South Africa) as our study primarily consisted of mixed-race youth and there were baseline race differences in our study. However, our findings were the same when the analyses were restricted to only mixedrace youth. Furthermore, while we were underpowered for analyses with only White or only Black youth due to the small number of each in our sample, the direction of effects was similar for these subsamples.

Our study findings should also be interpreted with caution as there were additional baseline differences for age and family income between the intervention and control groups, despite random assignment. We attempted to address these baseline differences by controlling for these factors as covariates in all our analyses. We were unable to include race as a covariate due to collinearity and the lack of Black and White youth in our study. In interpreting the findings of this study, it is helpful to keep in mind that lower socioeconomic status and being older are associated with earlier sexual debut, being older is associated with greater substance use, and there is a mix of findings with regard to the link between socioeconomic status and substance use (Hanson \& Chen, 2007; Lammers, Ireland, Resnick, \& Blum, 2000; Merikangas et al., 2010). For example, in a review by Hanson and Chen (2007) socioeconomic status was linked to adolescents smoking cigarettes but not to their alcohol and marijuana use. Thus, it is notable that HealthWise had significant impacts despite HealthWise students being poorer and older in comparison to students in the control condition.

Our findings are also limited by our measures, which are relatively new in the way we conceptualized activity reasons and are based on a small set of items for the mediators and outcomes. Nevertheless, this is a relatively unique method of assessing specific reasons for participating in specific activities in a large, longitudinal study. Thus, given our findings, developing further measures to unpack leisure activity motives and their link to positive youth development may be a promising area of research. We also focused on three of the most prevalent leisure activities among our sample. Spending time with friends, playing sports, and going to parks may not be as common in other populations or have the same quality and associated risks in other populations (e.g., while parks in this study were characterized as empty lots with frequent gang/drug activity, this is likely not as true for parks in suburban U.S. communities). Nevertheless, focusing on NED with friends, NED sports, and NED parks provided some insight into how reasons for participating in activities are influenced by an intervention and influence risky behavior.

Last, it is important to note that these findings are based on the experiences of youth between to 2004 and 2008. Although the increased use of technology since 2008 may take away from how much time youth spend doing activities such as those discussed in this article, the association between risky behaviors and youth engaging in NED activities is still 
likely relevant globally. For example, Biolcati, Mancini, and Trombini (2018) found greater boredom, use of technology, and alcohol use were all linked in a survey of Italian adolescents in 2015. Furthermore, in our experience in South Africa, the leisure activities described in this article are all still omnipresent every year since the current study. For example, many adolescent boys can still be found hanging out in empty parks with seemingly nothing else to do and engaging in risky behaviors.

Overall, despite these limitations, this study supports a leisure activity, context, and experience model (Caldwell, \& Faulk, 2013) and indicates a school-based prevention program can reduce risky behaviors among youth in low-income, underresourced areas by targeting leisure motives and specific activities for specific genders.

\section{Acknowledgments}

Appreciation is expressed to the HealthWise project staff and the schools, teachers, and youth who participated in this project.

\section{Funding}

This work was supported by the National Institute on Drug Abuse [grant number Ro1 DA01749 to the second author]. The first author was supported by the Institute of Education Sciences [grant number R305B090007] and by the National Institute on Drug Abuse [grant numbers T32 DA017629, P50 DA10075, P50 DA039838]. The views expressed in this article are ours and do not necessarily represent the official views of granting agencies. 


\section{References}

Bandura, A. (2001). Social cognitive theory: An agentic perspective. Annual Review of Psychology, 52(1), 1-26. doi:10.1146/annurev.psych.52.1.1

Biolcati, R., Mancini, G., \& Trombini, E. (2018). Proneness to boredom and risk behaviors during adolescents' free time. Psychological Reports, 121(2), 303-323. doi:10.1177/0033294117724447

Botvin, G. J., \& Griffin, K. W. (2004). Life skills training: Empirical findings and future directions. Journal of Primary Prevention, 25(2), 211-232. doi:10.1023/B:JOPP.0000042391.58573.5b

Caldwell, L. L., Bradley, S., \& Coffman, D. (2009). A person-centered approach to individualizing a school-based universal preventive intervention. The American Journal of Drug and Alcohol Abuse, 35(4), 214-219. doi:10.1080/00952990903005932

Caldwell, L. L., Darling, N., Payne, L., \& Dowdy, B. (1999). “Why are you bored?” An examination of psychological and social control causes of boredom among adolescents. Journal of Leisure Research, 31(2), 103-121. doi:10.1080/00222216.1999.11949853

Caldwell, L. L., \& Faulk, M. T. (2013). Adolescent leisure from a developmental and prevention perspective. In T. Freire (Ed.), Positive leisure science: From subjective experience to social contexts (pp. 41-60). New York, NY: Springer. doi:10.1007/978-94-007-5058-6_3

Caldwell, L. L., Patrick, M. E., Smith, E. A., Palen, L., \& Wegner, L. (2010). Influencing adolescent leisure motivation: Intervention effects of HealthWise South Africa. Journal of Leisure Research, 42(2), 203-220. doi:10.1080/00222216.2010.11950202

Caldwell, L., Smith, E., Wegner, L., Vergnani, T., Mpofu, E., Flisher, A. J., \& Mathews, C. (2004). HealthWise South Africa: Development of a life skills curriculum for young adults. World Leisure, 46(3), 4-17. doi:10.1080/04419057.2004.9674362

Collins, L. M., Schafer, J. L., \& Kam, C. M. (2001). A comparison of inclusive and restrictive strategies in modern missing data procedures. Psychological Methods, 6(4), 330-351. doi: 10.1037//1082-989X.6.4.330

Franklin, C., \& Corcoran, J. (2000). Preventing adolescent pregnancy: A review of programs and practices. Social Work, 45(1), 40-52. doi:10.1093/sw/45.1.40

Fredricks, J. A., \& Eccles, J. S. (2005). Developmental benefits of extracurricular involvement: Do peer characteristics mediate the link between activities and youth outcomes?. Journal of Youth and Adolescence, 34(6), 507-520. doi:10.1007/s10964005-8933-5

Graham, J. W., Palen, L. A., Smith, E. A., \& Caldwell, L. L., (2008). May). Attrition: MAR and MNAR missingness and estimation bias. Poster presented at the 16th Annual Meetings of the Society for Prevention Research, San Francisco, CA.

Hanson, M. D., \& Chen, E. (2007). Socioeconomic status and health behaviors in adolescence: A review of the literature. Journal of Behavioral Medicine, 30(3), 263285. doi:10.1007/s10865-007-9098-3 
International Narcotics Control Board (2013). Report of the International Narcotics Control Board or 2012. New York, NY: United Nations.

Kaufman, C. E., Clark, S., Manzini, N., \& May, J. (2004). Communities, opportunities, and adolescents' sexual behavior in KwaZulu-Natal, South Africa. Studies in Family Planning, 35, 261-274. doi:10.1111/j.0039-3665.2004.00030.x

Kumpfer, K. L., Smith, P., \& Summerhays, J. F. (2008). A wakeup call to the prevention field: Are prevention programs for substance use effective for girls?. Substance Use \& Misuse, 43, 978-1001. doi:10.1080/10826080801914261

Lammers, C., M., Ireland, M., Resnick, M., \& Blum, R. (2000). Influences on adolescents' decision to postpone onset of sexual intercourse: A survival analysis of virginity among youths aged 13 to 18 years. Journal of Adolescent Health, 26(1), 42-48. doi:10.1016/S1054-139X(99)oo041-5

MacKinnon, D. P., Lockwood, C. M., Hoffman, J. M., West, S. G., \& Sheets, V. (2002). A comparison of methods to test mediation and other intervening variable effects. Psychological Methods, 7(1), 83. doi:10.1037//1082-989X.7.1.83

Merikangas, K. R., He, J. P., Burstein, M., Swanson, S. A., Avenevoli, S., Cui, L., ... Swendsen, J. (2010). Lifetime prevalence of mental disorders in U.S. adolescents: Results from the National Comorbidity Survey Replication-Adolescent Supplement (NCS-A). Journal of the American Academy of Child and Adolescent Psychiatry, 49(10), 980989. doi:10.1016/j.jaac.2010.05.017

Motamedi, M., Caldwell, L. L., Wegner, L., Smith, E. A., \& Jones, D. (2016). Girls just want to know where to have fun: Preventing substance use initiation in an under resourced community in South Africa through HealthWise. Prevention Science, 17(6), 700-709. doi:10.1007/s11121-016-0654-3

Muth en, L. K., \& Muth en, B. O. (2010). Mplus user's guide (6th ed.). Los Angeles, CA: Muth en \& Muth en.

Ng, J. Y. Y., Ntoumanis, N., Thøgersen-Ntoumani, C., Deci, E. L., Ryan, R. M., Duda, J. L., \& Williams, G. C. (2012). Self-determination theory applied to health contexts: A metaanalysis. Perspectives on Psychological Science, 7(4), 325-340. doi:10.1177/1745691612447309

Palen, L. A., Patrick, M. E., Gleeson, S. L., Caldwell, L. L., Smith, E. A., Wegner, L., \& Flisher, A. J. (2010). Leisure constraints for adolescents in Cape Town, South Africa: A qualitative study. Leisure Sciences, 32(5), 434-452. doi:10.1080/01490400.2010.510975

Reddy, S. P., James, S., Sewpaul, R., Koopman, F., Funanl, N. I., Slfunda, S., \& Omardien, R. G. (2010). Umthente uhlaba usamila - The second South African youth risk behavior survey 2008. Cape Town, South Africa: South Africa Medical Research Council.

Rose, A. J., \& Rudolph, K. D. (2006). A review of sex differences in peer relationship processes: Potential trade-offs for the emotional and behavioral development of girls and boys. Psychological Bulletin, 132(1), 98-131.

Ryan, R. M., \& Deci, E. L. (2000). Self-determination theory and the facilitation of intrinsic motivation, social development, and well-being. American Psychologist, 55, 68-78. doi:10.1037/0033-2909.132.1.98 
Ryan, R. M., Patrick, H., Deci, E. L., \& Williams, G. C., (2008). Facilitating health behavior change and its maintenance: Interventions based on self-determination theory. The European Health Psychologist, 10, 2-5.

Seiffge-Krenke, I., (2011). Coping with relationship stressors: A decade review. Journal of Research on Adolescence, 21(1), 196-210. doi:10.1111/j.1532-7795.2010.00723.x

Sheldon, K. M., Ryan, R. M., Deci, E. L., \& Kasser, T. (2004). The independent effects of goal contents and motives on well-being: It's both what you pursue and why you pursue it. Personality and Social Psychology Bulletin, 30, 475-486. doi:10.1177/0146167203261883

Shilubane, H. N., Ruiter, R. A., C., Bos, A. E., R., van den Borne, B., James, S., \& Reddy, P. S., (2014). Psychosocial correlates of suicidal ideation in rural South African adolescents. Child Psychiatry \& Human Development, 45(2), 153-162. doi:10.1007/s10578-013-0387-5

Silbereisen, R. K., Eyferth, K., \& Rudinger, G. (Eds.) (1986). Development as action in context: Problem behavior and normal youth development. Berlin, Germany: Springer.

Skiba, D., Monroe, J., \& Wodarski, J. S., (2004). Adolescent substance use: Reviewing the effectiveness of prevention strategies. Social Work, 49(3), 343-353. doi:10.1093/sw/49.3.343

Smith, E. A., Palen, L.-A., Caldwell, L. L., Flisher, A. J., Graham, J. W., Mathews, C., ... Vergnani, T., (2008). Substance use and sexual risk prevention in Cape Town, South Africa: An evaluation of the HealthWise program. Prevention Science, 9(4), 311-321. doi:10.1007/s11121-008-0103-z

Tibbits, M. K., Smith, E. A., Caldwell, L. L., \& Flisher, A. J., (2011). Impact of HealthWise South Africa on polydrug use and high-risk sexual behavior. Health Education Research, 26(4), 653-663. doi:10.1093/her/cyro24

Townsend, L., Flisher, A. J., Chikobvu, P., Lombard, C., \& King, G., (2008). The relationship between bullying behaviours and high school dropout in Cape Town, South Africa. South African Journal of Psychology, 38(1), 21-32. doi:10.1177/008124630803800102

Wegner, L., (2011). Through the lens of a peer: Understanding leisure boredom and risk behaviour in adolescence. Journal of Occupational Therapy, 41, 18-24.

Wegner, L., Flisher, A. J., Chikobvu, P., Lombard, C., \& King, G., (2008). Leisure boredom and high school dropout in Cape Town, South Africa. Journal of Adolescence, 31(3), 421-431. doi: 10.1016/j.adolescence.2007.09.004

Weybright, E. H., Caldwell, L. L., Ram, N., Smith, E. A., \& Wegner, L., (2015). Boredom prone or nothing to do? Distinguishing between state and trait leisure boredom and its association with substance use in South African adolescents. Leisure Sciences, 37(4), 311-331. doi:10.1080/01490400.2015.1014530

Weybright, E. H., Caldwell, L. L., Xie, H. (J. )., Wegner, L., \& Smith, E. A., (2017). Predicting secondary school dropout among South African adolescents: A survival analysis approach. South African Journal of Education, 37(2), 1-11. doi:10.1570o/saje.v37n2a1353 